\title{
Linguistic Regularities of LOLspeak
}

\author{
Beata Bury \\ Philological School of Higher Education, Wrocław, Poland
}

\author{
Adam Wojtaszek \\ University of Silesia, Katowice, Poland
}

\begin{abstract}
The influence of the Internet on language is an unprecedented phenomenon. Maybe only the emergence of the systems of writing and the invention of printing can be claimed to have contributed more to the way language is used and perceived. Numerous publications have been devoted to the new forms of language thriving in the cyberspace. The present study focuses on one of the countless micro-genres which we may encounter while surfing the web: the LOLspeak. Its major peculiarity lies in the deliberate departure from the standard conventions related to grammar and orthography. At first sight, this new code of communication could puzzle and put off an average user, but upon closer inspection it turns out that there is some order in this apparent chaos and that there are certain limits to the deviations applied by ardent followers of LOLspeak. Our major aim was to propose a preliminary list of certain regularities characterizing this new form of linguistic creativity. It is by no means exhaustive, as the investigation is based on a relatively small corpus, but it suggests a certain direction for the future investigation of this very interesting phenomenon.
\end{abstract}

Keywords: LOLspeak, linguistic deviation, Internet language, linguistic innovativeness

\section{Introduction}

In the past, a narrow circle of experts, such as programmers, technicians, and designers had access to the computer and the Internet. In the course of time, the number of the Internet users has extended. Nowadays, the Internet provides a vast range of services, such as online shopping, daily news, religious practices, dating rooms, or journey planning, to mention but a few. Netizens communicate with each other through e-mails, forums, blogs, tweets, or chat rooms. As a result of that communication, new language forms, referred to as netspeak, netlish, weblish, or LOLspeak, have emerged. Numerous publications on the language of the Internet describe it as a new, hybrid form of language, combining the elements of speech and writing in an attempt to take the full advantage of the technical solutions offered by the quickly developing and evolving medium (e.g., Crystal, 2006; Wojtaszek, 2007). However, some of the new language forms encountered on the Internet nowadays are so surprising and unexpected that a question about the possible limits of inventiveness is raised.

The first appearance of LOLcats, i.e., funny images of macro cats with headings written in grammatically incorrect English known as LOLspeak, was on the 4chan website. Users posted pictures of cats with peculiar misspellings and simplified words there which, thanks to the 4chan website, made these cat macros and LOLspeak very popular. First attempts of examining LOLspeak were made by Calka (2011) and Miltner (2011), who did it in connection with their analysis of the LOLspeak community. Neither for Calka nor for Miltner was

Beata Bury, Ph.D. student, M.A., Faculty of Modern Languages and Literature, Philological School of Higher Education in Wrocław.

Adam Wojtaszek, Professor, Assoc. Prof., Institute of English, Faculty of Philology, University of Silesia. 
LOLspeak their main point of focus, they were more concerned with the reasons why it was used. Miltner concludes, for example, that LOLcats became popular because they made interpersonal communication easier and led to the development of a large Internet community. She examined three LOLspeak groups: Cheezfrenz, Meme Geeks, and Casual Users. According to Miltner, it is wrong to think that LOLspeak is used only by young people. Miltner (2011) assumes that "the most ardent Cheefrenz tend to be older women" (p. 25).

\section{What Is LOLspeak?}

According to Herring (2012), LOLspeak is a language play, a variation of a standard language whose users make their messages decorative, humorous, and ambiguous. LOLspeakers use grammatically incorrect English in order to play with its rules. As Huizinga (1955) notices, play makes it possible to leave the real life and engage in an entertaining activity with its own rules. The playful aspect of language has also been scrutinized by Cook in Language Play, Language Learning (2000), where he describes play as an indispensable component of cognitive development alongside with the development of imagination. Cook (2000) claims that manipulation of language brings certain groups of netizens together, whereas others are forced apart (p. 63). In other words, those netizens who do not comprehend language play are excluded from the LOLspeak community. Cook agrees that, although language play may seem unimportant, it provides essential information on how people manipulate language for establishing and maintaining social relationships.

One of the first attempts to analyze LOLspeak was made by Anil Dash, a blogger, who named it "a kitty pidgin":

(...) I realize this isn't a creole but more likely a pidgin language, used to help cats talk to humans. And since "pidgin" is already a cutesy spelling of a mispronunciation, there doesn't seem to be any really cute way to rename it to reflect its uniqueness. "Kitty pidgin" might be the closest thing we have to a name for this new language. (Dash, 2007, para. 8)

LOLcat phenomenon also received attention from Mark Liberman (2007) at Language Log. Liberman referred to Anil Dash's idea of LOLcat, its linguistic properties and the fact that users might get LOLspeak wrong. Liberman (2007) likened LOLspeak to a kitty baby-talk used for soppy interactions.

Lauren Gawne and Jill Vaughan disagree with Dash's opinion, claiming that LOLspeak does not match the characteristics of a pidgin. According to them (2011), LOLspeak has emerged from a manipulation of English alone and thus cannot be called a pidgin (p. 103), which is always a result of a contact between two languages.

LOLspeak attracted also the attention of Eduarda Abrahão de los Santos who compiled a glossary of LOLcat macros, i.e., pictures of cats with amusing captions which violate the conventional rules of spelling and grammar. For the purpose of her analysis, she used the website icanhascheezburger.com to select macros and then she translated them into orthographically correct English, e.g., sebben = seven, puhleeze = please (Santos, 2012, p. 73). Eduarda Abrahão de los Santos argues that LOLspeak should be given more attention from language professionals.

Aliza Rosen (2010), on the other hand, presents some linguistic variations present in LOLspeak and mentions the influence of other Internet forms on LOLspeak. She also attempts to formulate a workable definition by providing an answer to the question "whether it is a language unto itself, a dialect or variation of English, or something else" (Rosen, 2010, p. 7). Rosen used an illustration which involved translation of a passage about cats found at www.speaklolcat.com. In her conclusions, she states that LOLspeak bears no 
resemblance to Dash's "kitty pidgin". Rosen points out that pidgin is spoken, whereas LOLspeak has a written form. According to Rosen, LOLspeak resembles a mixture of baby talk and Leetspeak, the language used by game players.

Gawne and Vaughan, in turn, examined the material provided by the LOLcat Bible, a project aimed at translating the Bible into LOLspeak. They rejected the idea of describing LOLcats as "the stupidest creative act" (Shirky, 2010). According to them, LOLspeakers exhibit high English skills (Gawne \& Vaughan, 2012). They even believe that examining LOLspeak is vital to our understanding of language play and linguistic creativity (2012, p. 120).

The language play characteristics of LOLspeak are quite frequently explicitly referred to on the Internet by the users who engage in it:

(1) (a) Welkom tu dis happy playse. It is full of fun and silliness.

(Sir nativeca66, April 17, 2014, 5:08 p.m.)

(b) Come on over tu deh nooest LOL and play wif us!!

(cweenmj, April 17, 2014, 12:54 p.m.) ${ }^{1}$

As we can see, one of the distinctive features of LOLspeak is the departure from the standard spelling. The creativity of LOLspeakers can give the impression of extreme anarchy. It might seem, at the first sight, that the whole point is breaking all possible rules, but upon closer inspection it becomes quite clear that despite the linguistic variability and apparent chaos, certain grammatical patterns and regularities of LOLspeak can be enumerated.

\section{The Study}

The humour of LOLspeak's revolves around the concept of incongruity. An important ingredient is provided by photos of cats, which surprise the Internet user as they are somehow out of place. They usually involve a cat in one of its everyday poses, which behaves in a human way. The incongruity of the situation lies in the juxtaposition of an ordinary cat with the suggested human-like attributes. However, the present investigation does not focus on the cat images; instead, we attempt to grasp the linguistic peculiarities of the short comments which accompany them.

\section{Research Framework}

The existing linguistic analyses of LOLspeak focus on the exemplifications of grammatical and orthographic violations, accompanied by extensive "glossaries" of LOLspeak items with their translations to regular English, with the exception of Gawne and Vaughan's (2012) elaboration, which constitutes the first attempt to find patterns and regularities in the apparent chaos of LOLspeak. ${ }^{2}$ In our study, we were more interested in the limits to the modifications and violations applied by the LOLcat authors and in the extent to which variability is constrained in LOLspeak. In other words, not only what we find in LOLspeak is interesting, but also what is not there-what kinds of modifications would render comprehension of the texts too difficult or impossible.

In order to investigate the issue, we decided to compile a small corpus of 100 user comments placed on www.icanhascheezburger.com and www.yesterdazelolz.com websites, and to identify and describe some

\footnotetext{
${ }^{1}$ See http://www.yesterdazelolz.com/about-2/.

2 If we don't count the unpublished M.A. thesis by Jordan Lefler (2011).
} 
characteristic patterns of LOLspeak found there. The initial guide for the identification were the categories enumerated in Gawne and Vaughan (2012), but their extensive list was reduced to six items, where the limits of variability turned out to be easiest to pinpoint and describe. For this reason, the present study may be treated as a starting point for further investigation of more complicated patterns. The final list of categories includes the following: "CAN HAS" formula, auxiliary verbs, verb forms, manipulating categories, deviate spelling, and onomatopoeia.

Both sites were a rich source of LOLspeak lexicon and constructions. For convenience, the URLs of the examples quoted in the paper are provided in the footnotes. Because some of the comments or remarks placed on these websites, especially www.yesterdazelolz.com, turned out to be quite long, sometimes only relevant fragments were quoted.

\section{Research Results}

"CAN HAS" formula. The "CAN HAS" construction was popularized by Eric Nakagawa, one of the more influential LOLspeakers. Since the time it was first posted, the "CAN HAS" construction has been taken on by LOLspeak users all over the world and popularized on the Internet. Although it belongs, strictly speaking, to the more general category of Auxiliary verb manipulations, it is nevertheless presented separately as one of the most frequently found collocations. Some of the examples include:

(2) (a) Aye can has a gnu friend in Montrose, Colorado.

(Roofie, May 1, 2015, 1:47 a.m.) ${ }^{3}$

(b) (uploaded by Carl) ${ }^{4}$

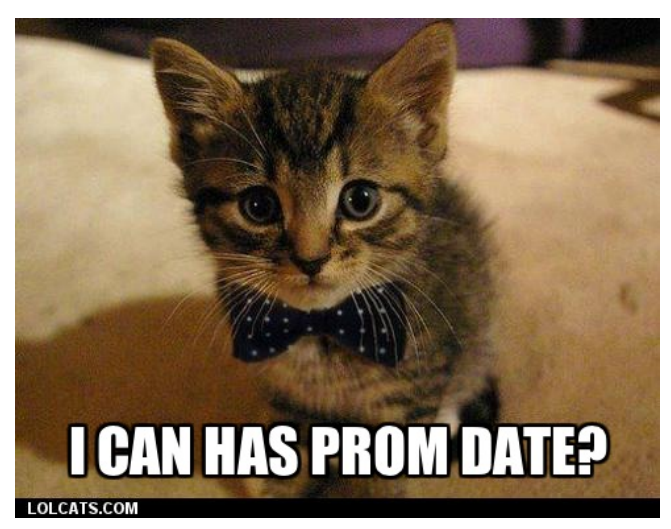

Two kinds of violations can be observed here. First of all, the inflected form of the main verb is placed after the modal auxiliary, instead of the infinitive. Additionally, however, the "CAN HAS" construction presents the variation in the word order, as it is used not only in statements, but also in questions. There is no subject-auxiliary inversion in (2b), although the question mark indicates that it is a question. In our data, this kind of inversion occurs frequently. The majority of LOLspeakers ( $60 \%$ of contributors) use the "CAN HAS" construction as the template to create their own funny captions. Interestingly, there were no examples where the modal verb "can" would be followed by the past tense form "had" or the participle form "having", which, theoretically, constitute two tempting alternations.

Auxiliary verb. LOLspeak is found to be a unique form of English which follows a deviant set of

\footnotetext{
${ }^{3}$ See http://www.yesterdazelolz.com/2015/04/30/dusty-is-convinced-hes-a-dog/.

${ }^{4}$ See http://www.lolcats.com/page-1.html.
} 
grammatical rules, including also syntactic patterns. LOLspeakers use sentence structures which diverge from Standard English. Since most of them involve alternative arrangements of the auxiliary constituent, they will be presented in the section titled "Auxiliary Verb". The most common variations are divided into several sub-categories presented below.

Question formation. In general, LOLspeak question formation is the same as in Standard English. LOLspeak users produce questions which follow the Standard English pattern:

(3) do yew come home to find giant blade swinging above door?

have yew fownd railway routed threw yowr kitchen?

has yowr life insurance papurs been examined lately?

was yowr holiday destinashun misteriusly changed frum Florida to bayroot?

(adorable smudgie, April 30, 2015, 4:53 p.m.) ${ }^{5}$

In those examples, LOLspeakers use appropriate verb forms to form questions. It shows that they exhibit a playful sophistication combined with a high level of grammar awareness. However, sometimes questions found in LOLcats do not follow the standard patterns, as illustrated below:

(4) AM dis whut Squidward luuked like azza babbeh?

(gunnersmama, February 1, 2016, 9:15 a.m.)

(5) Goggie snorin or da Missuss??

(Roofie, February 1, 2016, 6:03 a.m.) ${ }^{7}$

As we can see, in example (4) a wrong auxiliary verb form (1st person singular, instead of 3rd person singular) is used, whereas in example (5) the question status is only signaled by the punctuation mark at the end. Similarly to the "CAN HAS" construction in (2b), no inversion is applied in interrogative structures. The substitution illustrated in (4) only exemplifies the tendency, and actually involves many other verb form alternations, not just the verb "to be".

The modification which never occurs in the LOLcats is re-ordering of the structure of the question pattern: we have not found examples where the main verb would be fronted instead of or together with the auxiliary, or swapped in position with the auxiliary. The modifications are restricted to the patterns described above.

Auxiliary verb substitution. The tendency mentioned above is found not only in questions, but also in declarative constructions. It is quite frequent that LOLspeakers choose to replace the correct form of an auxiliary verb with another, as in the following examples:

(6) (a) Wicken cat am hiding inside

(Sirnativeca66, February 1, 2016, 3:35 p.m.) $^{8}$

I iz pritty

(turtlol, March 17, 2015, 9:08 p.m.) ${ }^{9}$

(b) da goggies hest steps awl ober eech

At leest her hazz only heds buy her hed

(Elsa Mama, February 1, 2016, 4:02 p.m.)

\footnotetext{
5 See http://www.yesterdazelolz.com/2015/04/30/who-would-have-known-my-human-could-read/.

${ }^{6}$ See http://www.yesterdazelolz.com/2016/02/01/youve-gotta-be-squiding-me/\#comment-112349.

7 See http://www.yesterdazelolz.com/2016/02/01/at-least-its-not-a-snake-pit/\#comment-112333.

8 See http://www.yesterdazelolz.com/2016/02/01/similar-yet-different/\#comment-112335.

9 See https://cheeztowncryer.wordpress.com/2015/03/14/kittyroo-am-gonna-walk-fru-deh-nite/\#comment-19497.
} 
tehy prolly hasta geddup wun atta tiem...

(cheshirekittehkat, February 1, 2016, 3:46 p.m.) $^{10}$

The most frequently applied alternation is the replacement of any of the forms of the verb "to be" with "am". If, however, the required form of the verb is the first person singular, then it is very readily replaced with an incorrect one. These consistently incorrect forms of the verb "to be" appear as one of the most prominent features of LOLspeak. As the examples in (6b) illustrate, also the verb "to have" in its auxiliary and main verb function is very readily altered, sometimes with forms non-existent in Standard English.

Although the forms of the auxiliaries are so variable, the auxiliaries themselves are very seldom used one instead of another, so we have not found the auxiliary "to be" used in place of "to have", or "to do" instead of "to have". The only exception is the auxiliary "to have" used instead of "to be" in predicative constructions.

Omission of auxiliary verb. Yet, in some cases the use of auxiliary verb is optional. The optionality of "do" or "have" appears mainly in interrogative and negative sentences.

(7) (a) It nawt maek yoo feel Poopular??

(Elsa Mama, March 29, 2014, 12:50 p.m.)

(b) How manee Morons yoo got burried ober der??

(Elsa Mama, April 6, 2014, 8:46 p.m.) ${ }^{11}$

The presence of question marks signals that the above statements are questions, while the main verb forms are indicators of the tense and aspect. Similarly to the above-illustrated auxiliaries, also the verb "to be" is omitted in interrogative and declarative sentences, usually in progressive constructions:

(8) (a) How YOO doin??

(Elsa Mama, April 30, 2015, 11:17 a.m.) ${ }^{12}$

(b) Butt aye nawt gettin off da wall.

(Elsa Mama, April 29, 2015, 8:25 p.m.) ${ }^{13}$

These omissions do not occur frequently when the verb "to be" functions as the copula verb, because then the clarity of the message would be significantly obscured.

Verb forms. To maintain the deliberate inflectional inconsistency, in LOLspeak, the main verb has usually the form of the third person singular in situations when the subject would require a different one, as the examples below show. All of them illustrate the lack of subject-verb agreement:

(9) (a) ai luvs re-reeding deze storees.

(dharma99, February 15, 2014, 10:52 a.m.)

(b) ai haz sumfin in mai eeys - scuse me.

(dharma99, February 15, 2014, 10:52 a.m.)

(c) Ai fanks u awl. Mai hart iz borked.

(kctailkinker, March 15, 2015, 7:38 p.m.)

\footnotetext{
${ }_{10}$ See http://www.yesterdazelolz.com/2016/02/01/at-least-its-not-a-snake-pit/\#comment-112359.

${ }_{11}$ See http://www.yesterdazelolz.com/submitting-a-lol/.

12 See

http://www.yesterdazelolz.com/2015/04/30/we-live-in-a-world-that-has-red-dots-and-those-dots-have-to-be-chased-by-fully-flo ofed-kitties/\#comment-73393.

13 See http://www.yesterdazelolz.com/2015/04/29/good-human-happy-human-paw-paw-paw/.
} 
(d) Am so sore ree $u$ nawt haz ur Inky bebbeh tu snorgling an pat-at-petting ennymoars an lubbing $u$ in purr son nawo.

(kat3kets1gog, April 19, 2015, 5:14 p.m.) ${ }^{14}$

This verb form is present in the first and the second person singular, for instance " $U$ still gots wun guud legg, rite?", but in our data we found that suffix -s was added to the verb in plural only twice. The suffix "-s" is present in both modal and ordinary verbs as in "sumday de reunions wills be glorious", and "dey weel unnerstands dey hafta nawt be chasin da kitteh", even in infinitives, for instance "Munniez iz nawt loose enuff tu gibs dis yeer". Lack of subject-verb agreement is a dominant LOLspeak regularity which appears in almost all analyzed comments $(92 \%)$. Characteristically, the modifications to the verb form do not include the "-ing" form used non-progressively: Wherever we find it on its own, it is always used in the progressive sense in constructions where the auxiliary is omitted.

As far as the past tense is concerned, LOLspeak users add suffix "-ed" to both regular and irregular verbs:

(10) (a) Grandma emptyed teh baskit!

(bluesfan473, May 1, 2015, 8:57 a.m.)

(b) Aye gotted held buy dat kewty pie cabana boi.

(Elsa Mama, April 30, 2015, 12:40 p.m.)

(c) Ai sed good bye and told him that he wuz da bestest kitteh.

(cweenmj, March 11, 2015)

It can be said that this LOLspeak phenomenon in some way reflects baby talk at a specific developmental stage, when children have the tendency to overgeneralize the newly discovered rules in their first language. Characteristically, however, with regular verbs, in order to maintain the deliberate incorrectness, the spelling conventions are violated, instead of attempting to invent forms based on some irregular patterns, e.g., "watch waught", on the basis of "catch - caught".

Manipulating categories. LOLspeak restructures the grammar of language and forms new configurations, playing with lexical categories. Often the distinction between nouns and adjectives is blurred:

(11) (images taken from www.cheezburger.com)

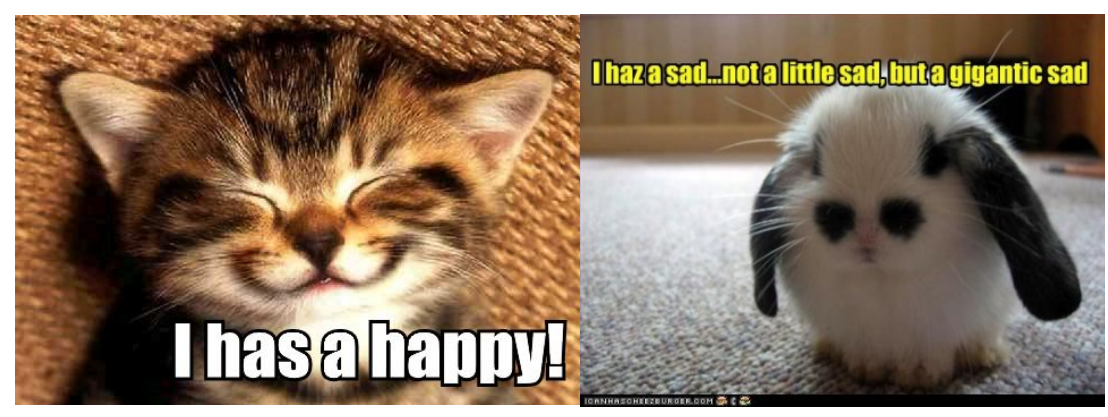

This change usually occurs with the verb "to have" (11) in the present and the past form, which in fact constitutes a replacement of the copula verb "to be" with "to have". The predicative construction normally requires an adjective in such situations, but the adjective is preceded with the indefinite article, so it acquires nominal features, and the subject predicative looks like a noun phrase. The interpretation could lead us to the reconstruction of the nominal head which has undergone ellipsis (probably the word "face"), which would

${ }^{14}$ See https://cheeztowncryer.wordpress.com/2015/03/11/inky-crosses-deh-bridge/\#comment-19495. 
justify the replacement of the auxiliary "to be" with "to have" and explain why this particular substitution is actually found in LOLcats. Alternatives such as "I does a happy" do not occur, because it would be too difficult to find a connection to the intended meaning.

Another lexical category change is illustrated by the word "gigantic", which in a modifying pre-adjectival position should be an adverb. The form of the adjective is, however, consistent with the nominal interpretation of the word "sad". Thus, correctness violated on one level is maintained on another. If "sad" is treated as a noun, then the category of the modifier is correct.

Because transcategorial conversion is not an infrequent phenomenon in Standard English, it is relatively easy to interpret words morphologically marked for one category as actually representing another in a phrasal combination. It is interesting, in this context, that adverbs are never used as noun modifiers, although correct interpretation of such erroneous combinations does not seem to pose a great mental challenge.

When it comes to the subcategorial shifts, LOLspeakers make uncountable nouns countable very often. The plural suffix is added to uncountable nouns as in "I gess wee duz have fun stuffs" or "Fur peeps wut nawt want to do munnies online". In LOLspeak, it is desirable to say "munnies" instead of "money"; this is by far the most dominant form encountered in the comments.

It can be said that LOLspeak users exploit language as a means of play and they manipulate it in a creative way to produce unique constructions. This obviously requires a high level of metalinguistic awareness and language instinct, as the examples involving the noun - adjective alternation have shown. However, it seems that the most prominent feature of LOLspeak is connected with the orthographic modifications.

Deviate spelling. Upon the first encounter with LOLspeak, the most striking feature seems to be the chaotic and inconsistent orthography. Almost every single word looks different from what it should and it is only thanks to relatively correct syntax and contextual knowledge that we are capable of deciphering the intended meaning. After some time, however, we notice that while LOLspeak users misspell words, they nevertheless follow certain rules and patterns, and there are visible limits of modifications being applied. The table below shows some regularities which can be discovered upon closer inspection. They most frequently involve a substitution of standard letter combinations with certain alternatives. The percentage value shows the ratio of comments in which a given change was found.

Table 1

Orthographic Regularities of LOLspeak

\begin{tabular}{|c|c|c|}
\hline Spelling change & LOLspeak & Standard English \\
\hline $\begin{array}{l}\mathrm{u}>-><\mathrm{OO}> \\
(90 \%)\end{array}$ & $\begin{array}{l}\text { inclooded (after a chase wich inclooded mee dragging her) } \\
\text { (poussinboi, April 30, 2015, 8:55 p.m.) }\end{array}$ & included \\
\hline $\begin{array}{l}<\mathrm{e} / \mathrm{ie}>-><\mathrm{ee}> \\
(67 \%)\end{array}$ & $\begin{array}{l}\text { mee (tehy'll be teh deth ov mee!!) } \\
\text { (poussinboi, April 30, 2015, 8:55 p.m.) }\end{array}$ & me \\
\hline & $\begin{array}{l}\text { laydees (Goelaydees!) } \\
\text { (bluesfan473, May 2, 2015, 5:11 p.m.) }\end{array}$ & ladies \\
\hline $\begin{array}{l}<\mathrm{v}>-><\mathrm{b}> \\
(67 \%)\end{array}$ & $\begin{array}{l}\text { hab(Aye'll hab salami) } \\
\text { (Elsa Mama, April 29, 2015, 10:18 p.m.) }\end{array}$ & have \\
\hline & $\begin{array}{l}\text { bisit (bisit wiffy mai kids furst Au Pair) } \\
\text { (Elsa Mama, May 1, 2015, 12:46 p.m.) }\end{array}$ & visit \\
\hline $\begin{array}{l}<\text { oul }>-><\mathrm{u}> \\
(10 \%)\end{array}$ & $\begin{array}{l}\text { wud (Sew, kitteh, wud yew laik sum noms...?) } \\
\text { (mantari, May 1, 2015, 7:42 p.m.) }\end{array}$ & would \\
\hline $\begin{array}{l}<0>-><\text { aw }> \\
(53 \%)\end{array}$ & $\begin{array}{l}\text { nawt (Deh comment race am nawt working) } \\
\text { (cweenmj, May 2, 2015, 5:07 p.m.) }\end{array}$ & not \\
\hline
\end{tabular}


(Table 1 continued)

\begin{tabular}{lll}
\hline Spelling change & LOLspeak & Standard English \\
\hline$<\mathrm{a}>-><$ ay $>$ & $\begin{array}{l}\text { laydees (Goe laydees!) } \\
(67 \%)\end{array}$ & ladies \\
$<\mathrm{x}>-><\mathrm{gg} / \mathrm{gs}>$ & neggst (git dere inn tyme fer neggst yeerz raise) & next \\
$(40 \%)$ & $\begin{array}{l}\text { (bluesfan473, May 3, 2015, 9:51 a.m.) } \\
\text { egspekted (PB egspekted tu hear teh gnus!!) }\end{array}$ & expected \\
& (poussinboi, May 2, 2015, 6:22 a.m.) & many \\
$<y>-><$ eh $>$ & menneh $^{15}$ & \\
$(63 \%)$ & & \\
\hline
\end{tabular}

As we can see, the changes are not drastic, and it is relatively easy to find a pattern. The replacements follow certain phonological dependencies: For example, a labiodental consonant "v" is replaced by a phonetically close bilabial consonant, "b", with which it also shares the feature of voicing. When it comes to the spelling of vowels, their incorrectness is based on possible spellings for the corresponding sounds in other orthographic configurations for English, for example the "-ay-" sequence which constitutes incorrect spelling in "laydees" is found in "day". Too distant alternative spellings are not employed, for example the spelling of the phoneme /f/ in "enough" never leads to misspelling of "of" as "ogh".

Another spelling pattern is the overuse of double vowel letters, for instance "hoomin" (human). Additionally, in LOLspeak, the vowel and consonant frequently switch their positions as in, for instance, "taht" (that) in "No, nawt as fat as taht wun" or "tihnk" in "Ai doan think killifying mee am tehir aim butt(!)", which are examples of such a manipulation.

The Table 1 above presents some regularities of forming LOL words. However, often the more frequently used words do not have just one but a number of different alternatives. An example is the verb "to have" which takes on various forms: "hab" in "Inky wz a lukki kitteh tu habs yu az a mawm", "habbing" in "Whai, kitteh, whut sleek fur yew habbing", "haz" in "ai haz sumfin in maieeys" or "hazz" in "At leest her hazz only heds buy her hed", with the correct form also appearing from time to time. The verb "to be" has its own variations, for instance, "I am" as in "Ai amm innersint" or "Ahm frum the gubbermint an ahm heer tew halp u". Another example is the pronoun "you" which is written as "yoo" as in "I likee yoo" or "yew" as in "Adn mae ai addink mai beenz fur yew tuu if yew oar yoarz am in knead?" or "yoou" as in "I hope Inky will come by to visit yoou from time to time". A further example includes "don't $t$ " as in "Naow goe awai an dunt bovver mee agin" or "Ai doan tihnk (...)". Such spelling variability may stem from the fact that the above words are used very frequently by LOLspeakers, but in positions where their correct interpretation does not pose a significant difficulty. It can be said that the more frequently the word is used, the more forms it possesses.

Examples (12) and (13) illustrate two ways in which the inventive orthography takes advantage of the existing word boundaries. In the first one, LOLspeak users tend to abbreviate certain phrasal expressions or frequent collocations into a single multi-syllabic word. The examples include "aifot" or "Ifinso" which appear in the first person singular:

(12) (a) Aifot (I thought) ai'd goe fur sumpfin subtlol, yet sproingy. (gunnersmama, May 2, 2015, 12:25 p.m.)

(b) Ifinso (I think so) I will wear dis wun. (cweenmj, May 2, 2015, 2:21 p.m.) ${ }^{16}$

\footnotetext{
${ }_{15}$ See http://www.yesterdazelolz.com/2015/04/30/best-use-practices-for-your-new-e-11 cat-blaster-rifle/.

${ }^{16} \mathrm{See}$ http://www.yesterdazelolz.com/2015/04/30/best-use-practices-for-your-new-e-11 cat-blaster-rifle/.
} 
The most frequently found blends involve the first person singular pronouns connected to the auxiliary verb or to the main verb, alternatively also in combination with the negative particle. The opposite tendency involves the division of longer multi-syllable words into smaller orthographic units, as in:

(13) (a) (...) Number Wun Sun in him's unib ersity [university] play.

(mantari, May 3, 2015, 5:59 a.m.)

(b) Ai mist all de egg site mint (excitement).

(lupinssupins, May 3, 2015, 3:57 a.m.) ${ }^{17}$

As we can see, sometimes the smaller items resemble existing words whose connected pronunciation is close to the divided unit, as in (13b), which very nicely demonstrates high metalinguistic awareness of the author.

Finally, LOLspeakers often propose alternative, although recognizable spelling of the less frequently used words, probably to attract the Internet users' attention and to provide a display of their skills:

(14) (a) Sew classy an elligunt tu!!!

(gunnersmama, May 2, 2015, 3:29 p.m.)

(b) whoo needs teh sentral heeting wen u gots such bootiful fur baybees fer a blankit?

(bluesfan743, February 1, 2016, 9:03 a.m.) ${ }^{18}$

Admittedly, all the linguistic changes of LOLspeak depend on the users' creativity, but also on their awareness of the existing limits. Through this language play, LOLspeakers demonstrate their high linguistic potential. They push linguistic boundaries as far as possible, but only within the limits of tolerable incongruity. The examples above also show that LOLspeakers use nonstandard language to achieve humorous effect. In Crystal's (2001) opinion, this kind of language play is what makes us human (pp. 222-225). Language play happens when the discourse rules are violated and language is used for fun. According to North (2007), humour becomes part of online interaction which cements the online community (p. 548). LOLspeakers leave their real-life willingly and engage in an online activity, establishing their own new principles. They become deliberately clumsy, infantile, playful, and creative.

It is often claimed nowadays that one of the prominent features of computer-mediated communication is overall recklessness when it comes to spelling rules. In LOLspeak, however, we have an impression that the users are in some way very attentive and careful about spelling, although in a very peculiar way. Here the major objective seems to be to violate the rules as a form of certain attitude manifestation. According to Sebba (2003), spelling variation is "the rebellion" against established norms and expression of independence (p. 168). He notices that netizens very often rebel against the standardized spelling rules, so LOLspeakers can be recognized as one of the peculiar subcultures within the Internet community.

Onomatopoeia and rhyming. LOLspeakers use onomatopoeic words to make the sentences more interesting and appealing to the reader. Imitation of sounds brings LOLspeak closer to spoken language and makes it more lively and emotional. Below there are some of the onomatopoeic words which LOLspeakers use:

(15) (a) *snap snap snap snap*

(Elsa Mama, May 5, 2015, 3:03 p.m.)

\footnotetext{
${ }_{17}$ See http://www.yesterdazelolz.com/2015/04/30/best-use-practices-for-your-new-e-11 cat-blaster-rifle/.

18 See http://www.yesterdazelolz.com/2016/02/01/at-least-its-not-a-snake-pit/\#comment-112339.
} 
(b) SKWEEEEEEEEEEEEEEE!!!!

(cweenmj, May 3, 2015, 4:02 p.m.)

(c) ...blargh...

(Roofie, May 3, 2015, 11:35 p.m.) ${ }^{19}$

Words "skwee" and "blargh" are used to express disgust with something. They indicate that LOLspeaker feels annoyance or anger toward a particular thing. Such expressions increase the emotional load of the message. Additionally, multiplication of letters in (15b), together with the use of capitals, symbolizes screaming.

Such a multiplication of letters is actually one form of another LOLspeak pattern: reduplication, which serves a variety of purposes, such as emphasis (16a), expression of happiness (16b), disgust (16d), or anger (16e). Some examples of LOLspeak reduplication include:

(16) (a) Buck am barkbarkbarkin at hiz hick ups.

(gunnersmama, May 3, 2015, 2:05 a.m.)

(b) *whappitahwhappitahwhappitah*

(bluesfan473, May 2, 2015, 10:42 a.m.)

(c) Am so sore ree u nawt haz ur Inky bebbeh tu snorgling an pat-at-petting ennymoars.

(kat3kets1gog, April 19, 2015, 5:14 p.m.)

(d) Oar teh sproingity wiskers!!!! skweeeeeeeeeeeeeeeeeeeeeeeee!!!

(bluesfan473, April 30, 2015, 11:19 a.m.)

(e) Urf, urf, urf! Errrr, gess dat shud b Arf, arf, arf.

(tortiemom77, March 31, 2015, 11:15 p.m.)

(f) Sum sooper dooper teecher (...)

(mantari, March 31, 2015, 9:04 p.m.)

(g) An' shii iz da cayootie patootie udderwize.

(Sir nativeca66, January 31, 2015, 11:55 p.m.) ${ }^{20}$

Examples above show that LOLspeakers use reduplication to create new words, to give special meaning to the word, to imitate sounds (16e) or copy the baby talk (Holm, 2011). In some cases, this is connected with the application of rhyming, which gives the comments a better rhythm and more attractive sound (16f-g). Thus, the departure from the standard in one dimension is counterbalanced by some expressive or rhythmical gain in another.

\section{Conclusion}

Similarly to other netizens, LOLspeakers alter and shape the text to show their originality and to impress the audience. It can be said that LOLspeak is "a display of communicative virtuosity" (Bauman, 2004, p. 9), where "performance flourishes" (Danet, 2001, p. 100). LOLspeakers construct the language of the group they want to be identified with and take part in the construction of meanings within it. They make use of linguistic resources available to them and adapt them to "their local level" (Eckert, 2003, p. 44). This innovative form of expression relies on language play, nonstandard use of grammar and altered sentence structure. As Gawne and Vaughan notice (2012), LOLspeakers take on identities of a cat and themselves. When they use LOLspeak as

\footnotetext{
19 See http://www.yesterdazelolz.com/2014/05/26/rainbow-skweeeeeeeeeeeeeeeeeeeeeeeeeeeee/.

${ }^{20}$ See http://www.yesterdazelolz.com/2014/08/14/8962/.
} 
cats, their language is playful and awkward. Yet, when they act as Internet users, their language is creative and exhibits metalinguistic awareness.

Due to the limited size of the corpus, in this paper we presented only selected aspects of LOLspeak, where the limitations to the apparent recklessness and irregularity are relatively easy to identify. We have managed to find a number of regularities within this language variety, such as consistent lack of subject-verb agreement, deliberate violations of verb morphology and moderate modifications of spelling, which involve most typically letter-reordering or letter sequence replacements. However, all these modifications are constrained to a high degree by the communicative purpose of LOLcats and their authors are careful not to go beyond certain limits. Several such restrictions have been identified on the basis of the analysis, which may constitute a starting point for further investigations of this intriguing language variety. Upon closer inspection, it turns out that LOLspeak is not a minor mode of communication but rather a very sophisticated language variety.

\section{References}

Bauman, R. (2004). A world of others' words: Cross-cultural perspectives on intertextuality. Malden, MA: Blackwell.

Calka, M. (2011). Language and ritual in Cheezland: The creation and maintenance of community in "I can has cheezburger" (Unpublished manuscript, Ohio University).

Cook, G. (2000). Language play, language learning. Oxford: Oxford University Press.

Crystal, D. (2001). Language play. Chicago: University of Chicago Press.

Crystal, D. (2006). Language and the Internet. Cambridge: Cambridge University Press.

Danet, B. (2001). Cyberplay: Communicating online. Oxford: Berg.

Dash, A. (2007). Cats can has grammar. In A blog on making a culture (April 23, 2007). Retrieved February 4, 2016 from http://anildash.com/2007/04/cats-can-has-gr.html

De los Santos, E. A. (2012). LOLcats and LOLspeak: The importance of the Internet culture for English professionals. Brazilian English Language Teaching Journal, 3(1), 62-76.

Eckert, P. (2003). The meaning of style. Proceedings of the eleventh annual symposium about language and society-Austin. Texas Linguistic Forum, 47, 41-53.

Gawne, L., \& Vaughan, J. (2012). I can haz language play: The construction of language and identity in LOLspeak. In M. P. L. Dao \& M. Bowler (Eds.), Proceedings of the 42nd ALS Conference-2011 (pp. 97-122). Canberra: Australian National University.

Herring, S. (2012). Special Internet language varieties: Culture, creativity \& language change. Paper presented at The II LETiSS Workshop Language Go Web: Standard and Nonstandard Languages on the Internet (April 4-5, 2012).

Holm, J. (2011). An introduction into pidgin and creoles. Cambridge: Cambridge University Press.

Huizinga, J. (1955). Homo ludens. A study of the play-element in culture. Boston, MA: Beacon Press.

Lefler, J. (2011). I can has thesis?: A linguistic analysis of LOLspeak (Unpublished M.A. thesis, Louisiana State University, Baton Rouge, LA). Retrieved from http://digitalcommons.lsu.edu/cgi/viewcontent.cgi?article=2270\&context=gradschool_theses

Liberman, M. (2007). Kitty pidgin and asymmetrical tail-wags. In Language log (April 25, 2007). Retrieved February 4, 2016 from http://itre.cis.upenn.edu/ myl/languagelog/archives/004442.html

Miltner, K. (2011). Srsly phenomenal: An investigation into the appeal of LOLcats. London: London School of Economics and Political Science.

North, S. (2007). "The voices, the voices": Creativity in online conversation. Applied Linguistics, 28(4), 538-555.

Rosen, A. (2010). Iz in ur meme aminalizintehlangwich: A linguistic study of LOLcat. Verge, 7, 1-12.

Sebba, M. (2003). Spelling rebellion. In J. Androutsopoulos and A. Georgakopoulou (Eds.), Discourse constructions of youth identities (pp. 151-172). Amsterdam: John Benjamins.

Shirky, C. (2010). Cognitive surplus: Creativity and generosity in a connected age. London: Penguin.

Wojtaszek, A. (2007). Informatyczna e-wolucj@ języka w przestrzeni wirtualnej (IT e-volution of 1@nguage in cyperspace). In A. Łyda (Ed.), Przestrzenie języka (Dimensions of language) (pp. 151-170). Katowice: Wydawnictwo WSZMiJO. 